Béatrice Bouvier, Anne-Marie Châtelet, Sabine du Crest, François Fossier, Anne Georgeon-Liskenne, Itzhak Goldberg, Jean-Claude Golvin, Hélène Jannière, Jean-Michel Leniaud, Anne Péan et Pierre Rouillard

Actes du Ve

congrès national

d'histoire de l'art

Actes du Ve congrès national d'archéologie et d'histoire de l'art

Bordeaux, 21-24 octobre 1999

Publications de l'Institut national d'histoire de l'art

\title{
Le point de vue critique de la presse architecturale germanique sur l'architecture contemporaine française dans les années 1840-1914
}

\section{Anne Georgeon-Liskenne}

DOI : 10.4000/books.inha.2295

Éditeur : Publications de l'Institut national d'histoire de l'art

Lieu d'édition : Bordeaux

Année d'édition : 1999

Date de mise en ligne : 5 décembre 2017

Collection : Actes de colloques

ISBN électronique : 9782917902585

\section{Sbooks}

http://books.openedition.org

\section{Édition imprimée}

Date de publication : 24 octobre 1999

\section{Référence électronique}

GEORGEON-LISKENNE, Anne. Le point de vue critique de la presse architecturale germanique sur l'architecture contemporaine française dans les années 1840-1914 In : Actes du Ve congrès national d'archéologie et d'histoire de l'art : Bordeaux, 27-24 octobre 1999 [en ligne]. Bordeaux : Publications de I'Institut national d'histoire de l'art, 1999 (généré le 18 décembre 2020). Disponible sur Internet: <http://books.openedition.org/inha/2295>. ISBN : 9782917902585. DOI : https://doi.org/10.4000/ books.inha.2295. 


\title{
Le point de vue critique de la presse architecturale germanique sur l'architecture contemporaine française dans les années 1840-1914
}

\author{
Anne Georgeon-Liskenne
}

1 En Allemagne, la presse architecturale existe depuis 1789. En Autriche l'Allgemeine Bauzeitung (Revue générale de construction), revue d'importance nationale très ouverte aux créations étrangères, est fondée à Vienne en 1836. Jusqu'en 1914 des dizaines de titres sont créés dans les principales villes des pays de langue allemande pour répondre aux attentes d'un public spécialisé de constructeurs, ingénieurs, architectes, dispersé entre les provinces de l'Allemagne et de l'Empire austro-hongrois. Parler de presse germanique sur une période de soixante-quinze ans revient à évoquer les discours de plusieurs générations d'architectes plus ou moins marqués par la guerre francoprussienne et par l'émergence des nationalismes en Europe Centrale.

2 Dans un siècle passionné par la critique philosophique, esthétique ou littéraire, on peut se demander quels types de personnalités produisent la critique d'architecture, sous quels angles les architectes rédacteurs considèrent la France, et quels programmes architecturaux ils privilégient pour l'équipement des villes.

\section{Les critiques d'architecture}

3 La revue architecturale en pays allemands est l'organe d'une association rassemblant à la fois des architectes, des ingénieurs ou des constructeurs moins diplômés. Les rédacteurs font en général partie de ces associations qu'ils renseignent sur les développements de l'architecture à l'étranger. Comme la plupart des articles sur la France ont pour seul objectif celui d'informer, l'expression subjective de la critique est rarement de mise et les rédacteurs d'articles restent anonymes ou bien signent seulement de leurs initiales. 
4 Malheureusement pour le chercheur, il arrive que les auteurs des rares articles de fond soient difficiles à identifier. On ne sait rien de C.Jk., par exemple, sinon que ce rédacteur connaît bien la France et qu'il s'inspire entre 1882 et 1894 de deux revues françaises, la Gazette des architectes et du bâtiment et l'Architecture pour rédiger successivement dans la Deutsche Bauzeitung (Revue allemande de construction) une série d'études sur l'état de l'architecture en France : la réglementation urbaine à Paris, les architectes français devant les tribunaux, l'art à Berlin du point de vue français, les architectes français décédés dans les années 1880 et 1890, les progrès de la construction en France.

5 Quand l'auteur peut être identifié, on progresse beaucoup dans la compréhension du point de vue qu'il propose sur la France. En voici deux exemples avec Gottfried Semper (1803-1879) et Hubert Stier (1838-1907) : exilé d'Allemagne après le soulèvement de mai 1849, l'architecte de l'opéra de Dresde, Gottfried Semper, devient correspondant à Paris du mensuel Zeitschrift für praktische Baukunst (Journal de construction pratique) publié à Leipzig et Berlin. Familier des expositions et du milieu parisien, il propose une série d'articles sur l'art de la Seconde République, sur un immeuble de la rue Saint-Georges, ou sur la bibliothèque Sainte-Geneviève. Lui qui a toujours préféré dans son œuvre la fantaisie baroque, les formes plantureuses et les ressources de la polychromie, il juge la structure métallique apparente de la bibliothèque d'Henri Labrouste trop froide et sans monumentalité.

6 Hubert Stier, architecte originaire de Berlin, profite d'un voyage fait à travers la France en 1867 à l'occasion de l'exposition universelle pour exprimer dans la Deutsche Bauzeitung ses impressions sur l'état de l'architecture en France. Sa participation au congrès international des architectes, rapportée dans le Bulletin de la Société centrale des architectes de 1867, lui fournit un premier point de vue sur cette question. Il semble avoir été particulièrement marqué par ses échanges avec Eugène Emmanuel Viollet-leDuc lors d'une visite du château de Pierrefonds alors presque entièrement restauré. Malgré son enthousiasme pour l'enseignement du maître, il a passé sa carrière à édifier des monuments de style très éclectique (fig. 1). 
Fig. 1 Hubert Stier. Gare de Kreiensen édifiée en 1887-1889, dessin de l'entrée principale, Architektonische Rundschau, 1891, pl. 66.

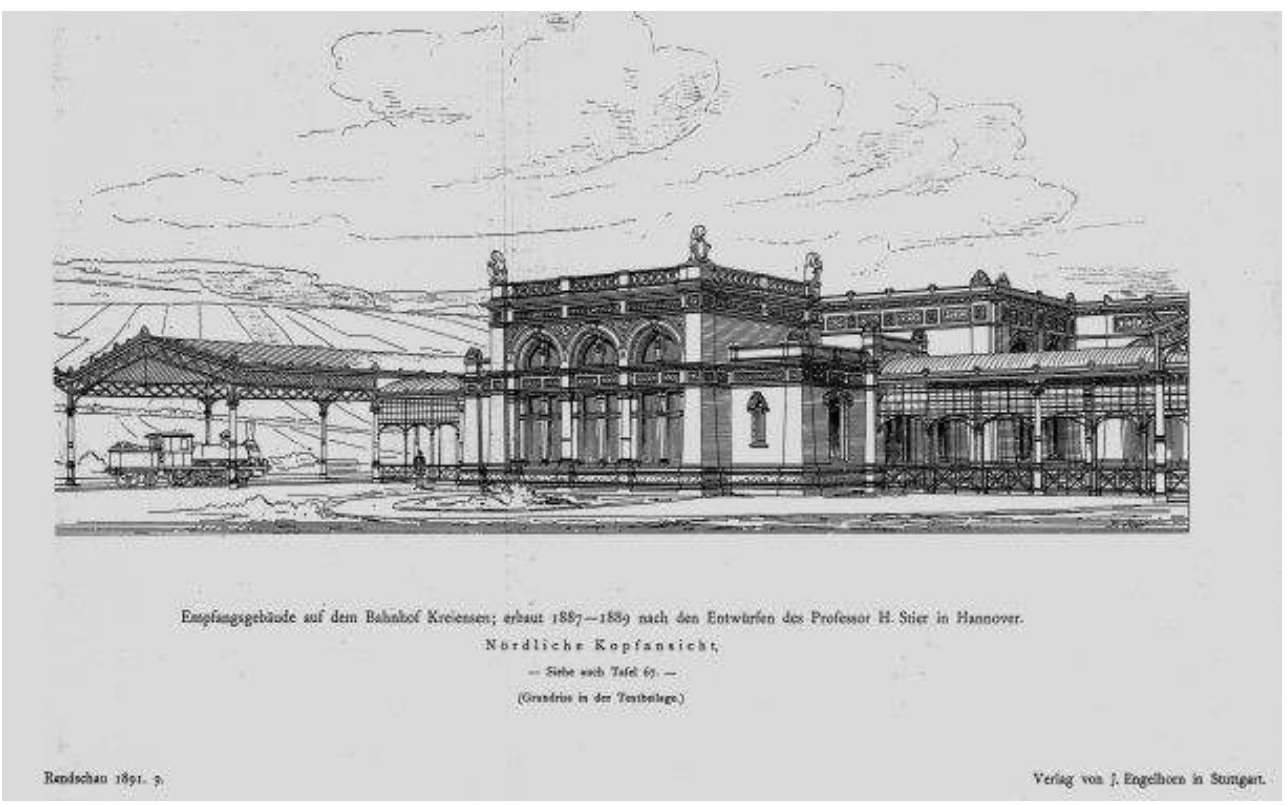

7 Dans un article paru en 1868 dans la Deutsche Bauzeitung, Hubert Stier adopte entièrement les arguments de Viollet-le-Duc contre l'intervention de l'État dans l'enseignement de l'art. Citant l'auteur de l'opuscule Ce que réclame au XIXe siècle l'enseignement de l'architecture, il écrit: "On ne viendra dans cette école [des beauxarts], non plus pour apprendre, mais pour passer des examens et ces examens que l'on passe seulement pour obtenir un grade ne sont ni un moyen de connaissance, ni la preuve d'une formation quelconque ». Il trouve les envois de Rome "ennuyeux et uniformes " dépourvus de «la variété et de la saine authenticité d'esquisses de voyage ». Ces travaux d'élèves pourtant brillants laissent une place fort réduite à la construction. Théorie et pratique lui semblent bien mieux accordées à l'École spéciale d'architecture fondée par Emile Trélat deux ans auparavant en 1865. Ce long article polémique n'a pas exercé une grande influence sur la fréquentation de l'École des beaux-arts par les étudiants allemands en architecture, mais le vent commence à tourner en faveur de la modernité germanique (enseignement de l'architecture, recherches stylistiques, connaissance de l'actualité architecturale étrangère), et la France apparait de moins en moins, particulièrement après la guerre de 1870, comme le mentor des architectes occidentaux.

\section{Les angles de la critique}

Les articles sur l'architecture française sont extrêmement variés et couvrent ainsi toute l'actualité architecturale, de la simple information à l'article de fond. Mais, pour pouvoir parler vraiment de critique, il faut sélectionner les articles les mieux documentés. Ils ne sont finalement pas si nombreux car la presse s'occupe moins de critique d'art que d'informations techniques ou générales sur l'organisation professionnelle ou la typologie des édifices contemporains en France. Les réflexions théoriques sur le choix du style, des matériaux, sur la recherche de la modernité qui 
animent les débats entre les architectes français et les divisent en plusieurs courants ne sont pas à ma connaissance diffusées au-delà des frontières.

9 J'ai distingué trois thèmes de prédilection dans les revues de langue allemande: les notices biographiques, les expositions universelles et les édifices français.

Les courtes biographies s'inspirent, en réalité, le plus souvent des nécrologies parues à la mort de l'artiste dans les périodiques spécialisés français. La nécrologie est un exercice qui se pratique dans tous les types de revues d'architecture germaniques, tant techniques que décoratives. Elle est le plus souvent un hommage rendu à de grands architectes français : Victor Baltard, dont les fameuses halles l'ont fait connaître outreRhin, Charles Auguste Questel qui eut beaucoup d'élèves suisses et allemands dans son atelier de l'École des beaux-arts, Henri Labrouste dont les travaux à la Bibliothèque nationale et la bibliothèque Sainte-Geneviève ont été diffusés dans l'Allgemeine Bauzeitung de Vienne par l'un de ses élèves autrichien Albrecht Rosengarten, Auguste Magne qui, à sa mort en 1885, est présenté comme un architecte «majeur » de théâtres, Théodore Ballu, un des architectes "les plus importants", auteur de l'église de la Trinité, "intéressante pour son utilisation du fer», ou encore, Charles Garnier. L'exception parmi ces noms connus reste au début du XXe siècle Léon Benouville, pourtant moins célèbre que ses contemporains Hector Guimard ou Henri Sauvage. Un mensuel de Stuttgart, les Moderne Bauformen, le présente en 1904 comme l'architecte du kleiner Mann, supérieur à ses élégants camarades Plumet et Selmesheim ou même à l'imaginatif Guimard pour son grand naturel et son style adapté aux gens simples.

11 Mais il reste un architecte dont la carrière et l'œuvre ont été connus avant et encore plusieurs décennies après sa mort, phénomène rare dans la presse : c'est Viollet-le-Duc, dont les écrits et les dessins sont diffusés dans tous les pays de langue allemande. "Aussi longtemps qu'on apprendra à assembler des pierres entre elles pour construire des temples, des palais, des maisons, on parlera de Viollet-le-Duc", écrit Arthur Baignières en 1881, deux ans après la mort du maître, dans un journal d'arts décoratifs publié à Leipzig, la Zeitschrift für bildende Kunst. Jamais un architecte français n'avait fait l'objet d'un article aussi long ni aussi laudateur. À la lecture de ces dix pages, on se demande si l'auteur a pris en compte tous les aspects du travail de l'architecte restaurateur. Tout semble réussi à en croire Arthur Baignières : « Viollet-le-Duc a rendu la vie à Pierrefonds", "toutes ses restaurations révèlent sa finesse et la profonde connaissance qu'il avait de l'esprit des maîtres du Moyen Âge roman et gothique ». Mais rien n'est dit sur le rationalisme, l'utilisation vraie des matériaux; seul l'archéologue est mis en valeur et les quelques réalisations personnelles de l'architecte ne sont pas évoquées. C'est avec plus d'esprit critique que la Deutsche Bauzeitung prussienne avait dénoncé le «système » de l'auteur du Dictionnaire dans ses nombreux articles. Cinq ans après la mort de l'architecte français, un rédacteur connu sous les initiales E.E.E., commentant le livre d'Anthyme Saint-Paul sur Viollet-le-Duc, ses travaux d'art et son système archéologique, qualifie de «roman d'architecture» l'œuvre écrit du restaurateur. Tout l'article dénonce la suffisance de l'architecte et son esprit doctrinaire qui fit passer « sur le lit de Procuste » les contradictions de son système. Cet auteur allemand est dans la lignée de John Ruskin et de Wilhelm Lübke qui dénonçait le "vandalisme restaurateur » des architectes, mais il annonce aussi le débat engagé par Georg Dehio et l'Autrichien Alois Riegl sur le monument à la fin du siècle. Ainsi, comme en France, c'est surtout autour du nom de Viollet-le-Duc que la critique germanique 
s'affine, s'aiguise, s'anime et prend position car elle est, en définitive, presque toujours polie, voire policée, plutôt urbaine.

L'œuvre de Viollet-le-Duc est en outre particulièrement bien diffusé par les dessins que la commission des monuments historiques présente dans les expositions universelles. Le rôle de ces foires internationales est essentiel pour mesurer l'ampleur des échanges entre la France et les pays allemands. Si on trace la courbe du nombre d'articles parus sur la France durant la période, on voit aisément les pics des années 1867, 1878, 1889 et 1900, dates des expositions universelles à Paris (fig. 2, 3, 4 et 5). C'est parfois le seul sujet qui permette de centrer l'attention sur la France dans des revues spécialisées comme la Zeitschrift für Bauwesen, organe du ministère des travaux publics de Berlin, ou la Zeitschrift für Baukunde publiée à Munich. Ce «concours pacifique de la culture ", confrontation aimable des intelligences entre les pays dans un contexte de tensions politiques, suscite beaucoup d'espoirs en la civilisation et la paix. Les réflexions de l'ingénieur L.A. Barré dans la Revue générale de l'architecture et des travaux publics sont représentatives de ces espoirs. Les possibilités techniques de destruction sont telles, écrit-il, qu'elles enlèveront toute envie aux hommes de faire la guerre. Mais l'enthousiasme manifesté en 1867 est étouffé par la guerre francoprussienne de 1870 et par l'alliance autrichienne avec l'Empire allemand unifié. Le ton de la presse germanique change: le point de vue critique des architectes se mêle de considérations politiques. Une pointe de francophobie est perceptible en 1878 et plus encore en 1889 lors du centenaire de la Révolution.

Fig. 2 Articles sur la France dans 30 revues allemandes dépouillées sur 60 ans (1855-1914).

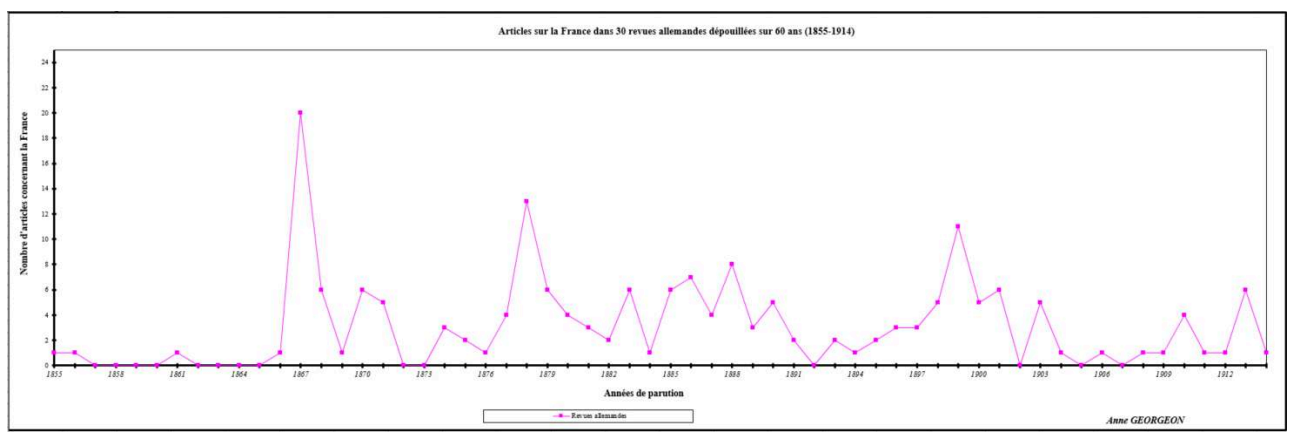

Fig. 3 Articles sur la France dans 18 revues austro-hongroises dépouillées sur 72 ans (1843-1914).

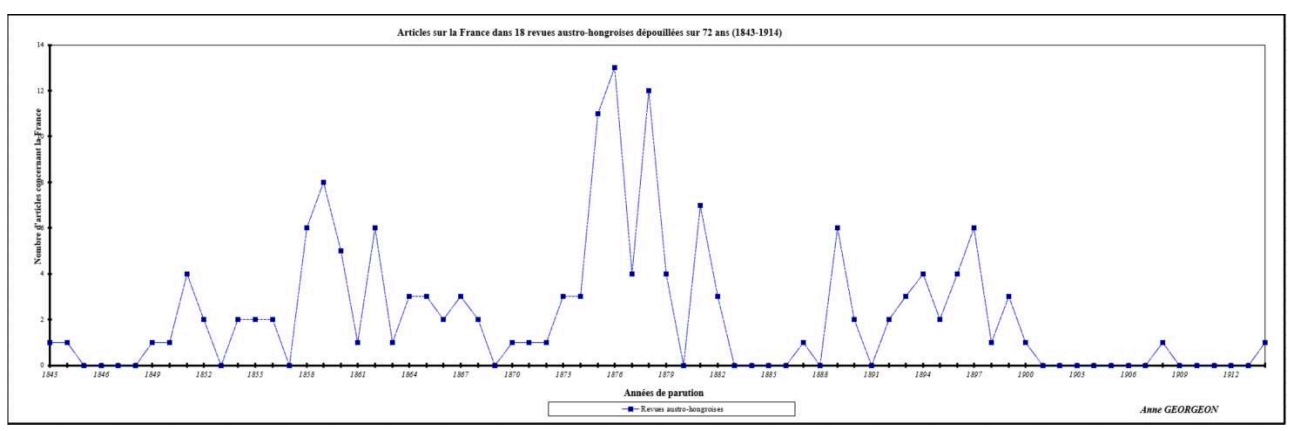


Fig. 4 Articles sur la France dans 48 revues allemandes et austro-hongroises dépouillées sur 72 ans (1843-1914).

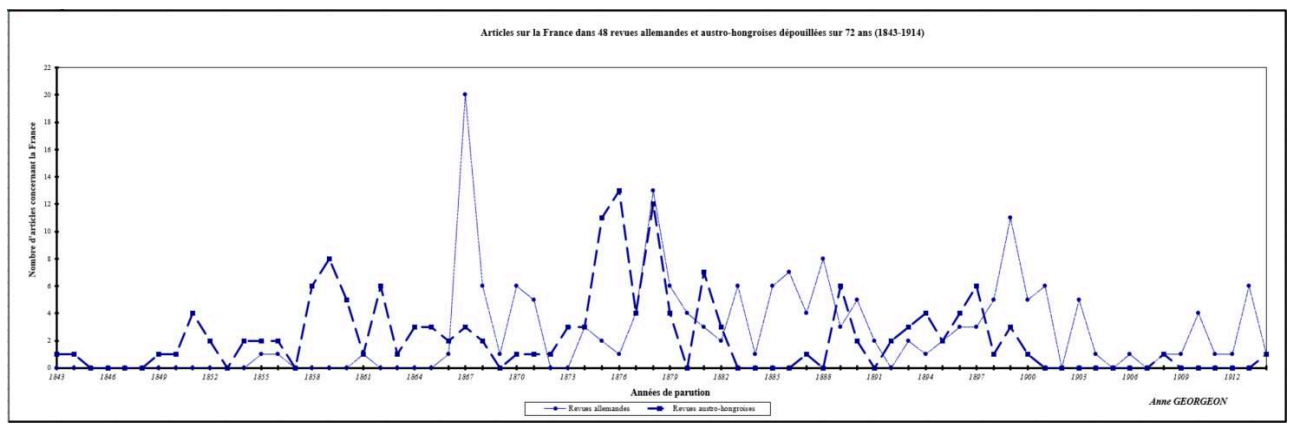

Fig. 5 Somme des articles sur la France parus dans 48 revues allemandes et austro-hongroises dépouillées sur 72 ans (1843-1914).

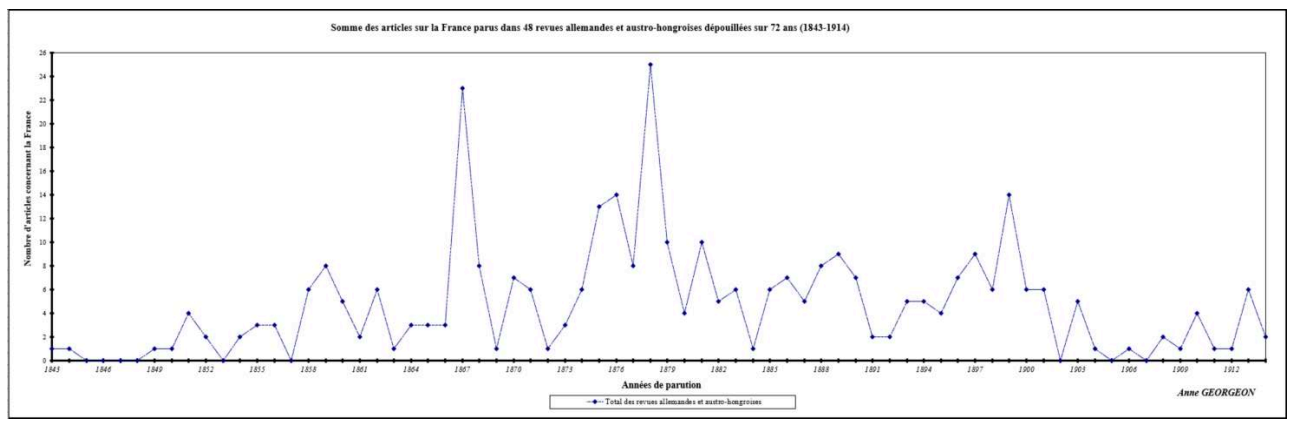

L'évolution du ton est bien plus sensible dans les articles sur les œuvres architecturales que dans ceux qui portent sur les caractères techniques des bâtiments. L'Allemand Jakob von Falke, auteur de nombreux ouvrages théoriques sur les arts appliqués, encourage les artistes à engager "une réforme dans une direction antifrançaise " comme il l'écrit à l'occasion de l'exposition universelle de Vienne en 1873. La France présente en effet depuis plusieurs années les mêmes décorations d'habitation, alors que l'Angleterre et l'Autriche se tournent vers le style oriental. La France pourtant a obtenu cette année-là le nombre le plus important de médailles dans la section architecture. Mais les rédacteurs français sont les seuls à le souligner. Ils ne cachent pas non plus que leurs rivaux progressent, d'une part grâce à l'essor de l'industrie dans l'Empire allemand, d'autre part, grâce à la florissante Académie de Vienne qui forme jusqu'au tournant du siècle les architectes du Ring et de la modernité viennoise.

Lors de l'exposition universelle de 1889, la France apparaît dans les articles de la Deutsche Bauzeitung comme un pays trop vieux, qui s'est isolé et qui manque d'idées. Finies les louanges envers la patrie du bon goût. La tour Eiffel apparaît comme un " malheur esthétique » et, en 1900, quand la fête de l'art nouveau bat son plein, la France poursuit ses "compliments décoratifs aux styles du passé ». Le Grand Palais dont l'architecte a fait un compromis entre une colonnade Louis XVI et une verrière mesquine aux arcs de fer trop nus irrite les rédacteurs du périodique Der Architekt, revue fondée en 1895 à Vienne et très favorable à Otto Wagner.

Mais autour des expositions universelles dont les bâtiments éphémères représentent le goût français, toute l'architecture parisienne et durable trouve davantage grâce aux yeux de ses adversaires. La critique étrangère s'exerce en effet régulièrement sur les édifices de la capitale. 

apparaître une légère augmentation autour des dates de grands chantiers comme l'Opéra de Charles Garnier. Pourtant, en général, les parutions se répartissent de manière homogène. Il me semble qu'on peut les diviser en trois rubriques. La première comporte les ouvrages très techniques, les usines comme celle de Meunier à Noisiel que l'on retrouve dans deux revues, les gares, les halles métalliques, les passages couverts, les ouvrages d'art. La deuxième est celle des nouveautés, qui sont l'objet de courts articles, notamment les maisons et villas particulières, immeubles, le théâtre du Vaudeville, le nouvel Hôtel-Dieu, la prison de la Santé, l'asile de Vincennes, l'église Sainte-Clotilde et de rares exemples en province. Enfin, les chantiers spectaculaires de la période fournissent des modèles aux rédacteurs architectes : l'Opéra de Paris et les grands magasins en sont les deux principaux exemples.

Dans les nombreux articles sur les constructions françaises destinées au public, opéras, théâtres, grands magasins, hôpitaux, un thème revient comme un signal d'alarme : le manque de sécurité en matière d'incendie et d'hygiène. C'est la critique la plus virulente et la plus récurrente. En 1887, année de l'incendie de l'Opéra comique, le Wochenblatt für Baukunde (Hebdomadaire des sciences de la construction) rappelle que les salles parisiennes sont moins sûres que celles de Munich ou de Vienne parce qu'elles ne sont pas exclusivement éclairées à l'électricité. En 1900, une semaine après l'incendie du Théâtre français, un rédacteur allemand conclut que « la loi française ne connaît pas les sévères prescriptions que suivent l'Allemagne et l'Autriche pour la construction des théâtres ».

Un édifice trouve néanmoins grâce aux yeux des critiques allemands, l'Opéra de Paris, dans lequel Charles Garnier a aménagé des galeries d'évacuation pour le public et le personnel, disposé des escaliers à double hélice, introduit l'électricité et des câbles métalliques. Les seuls reproches techniques que l'on rencontre touchent la ventilation défectueuse et la chaleur étouffante. Cet édifice, qui n'a quasiment pas été étudié à l'époque par les revues spécialisées françaises, est néanmoins bien connu des rédacteurs étrangers. Cette observation laisse supposer que les revues françaises n'étaient pas le seul moyen pour nos voisins de s'informer sur l'activité édilitaire en France, et qu'il existait d'autres relais comme des "correspondants sur place", qui devaient communiquer aux revues professionnelles le fruit de leur visite en France.

Après la réalisation de l'opéra, l'œuvre de Charles Garnier trouve régulièrement un écho dans la presse allemande, comme son Panorama des Champs-Élysées, celui de la rue Saint-Honoré ou l'hôtel du Cercle de la librairie boulevard Saint-Germain (fig.6). 
Fig. 6 Charles Garnier, Panorama des Champs-Elysées, Cercle de la Librairie, Panorama de la rue Saint-Honoré, Deutsche Bauzeitung, 1887, p. 29.

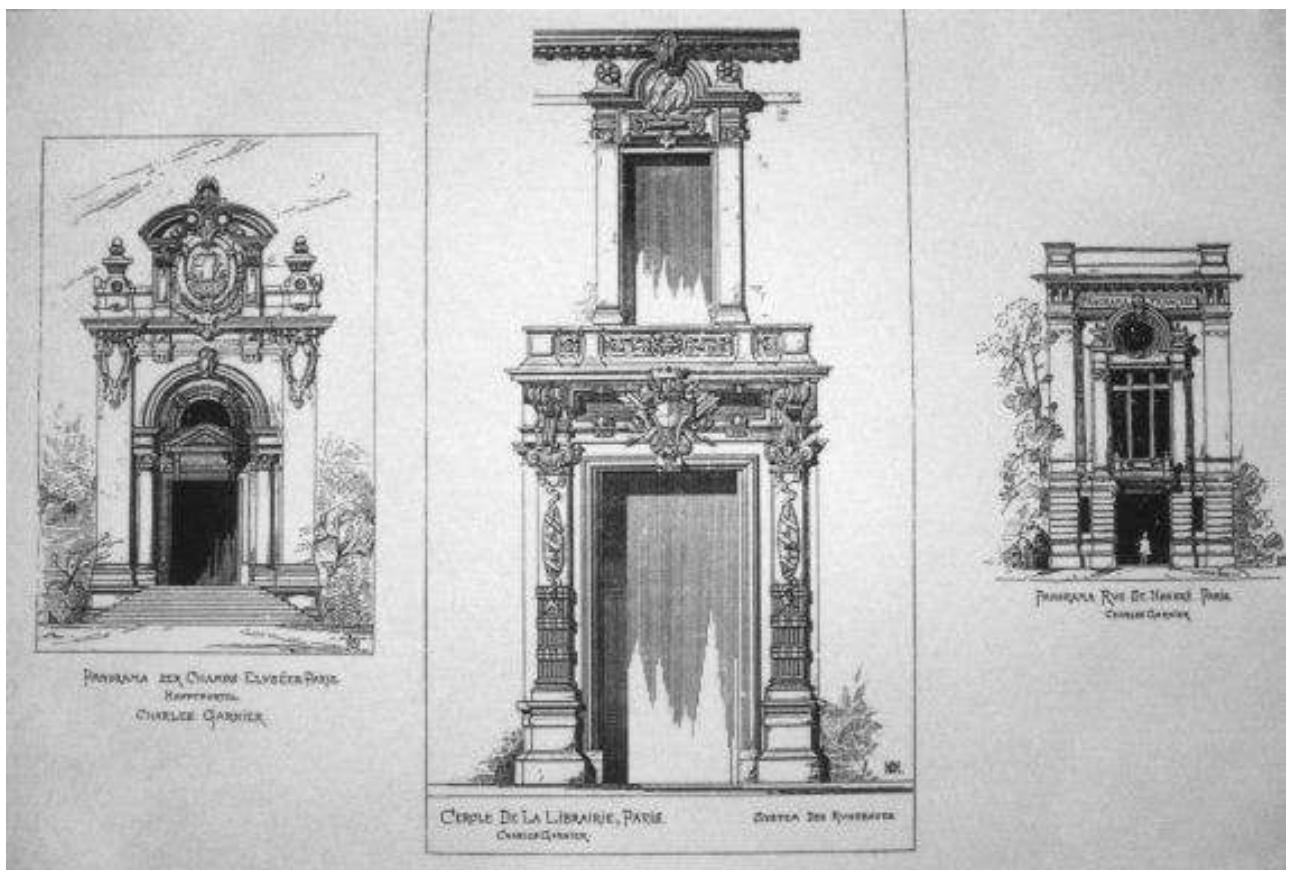

21 Sous l'angle du style, l'Opéra Garnier a séduit ses contemporains. Plusieurs qualificatifs reviennent sous la plume des rédacteurs allemands, autrichiens, tchèques ou hongrois : ceux de fastueux (prunkhaft), heureux (glücklich), puissant (mächtig), riche. La Budapester Bauzeitung en fait la une de son numéro d'octobre 1875 en le comparant avantageusement avec son contemporain, l'opéra de Vienne (fig. 7). 
Fig. 7 -« Die grosse Oper in Paris » de Ch. Garnier, Budapester Bauzeitung, 17 octobre 1875, article extrait de la Baugewerkzeitung allemande.

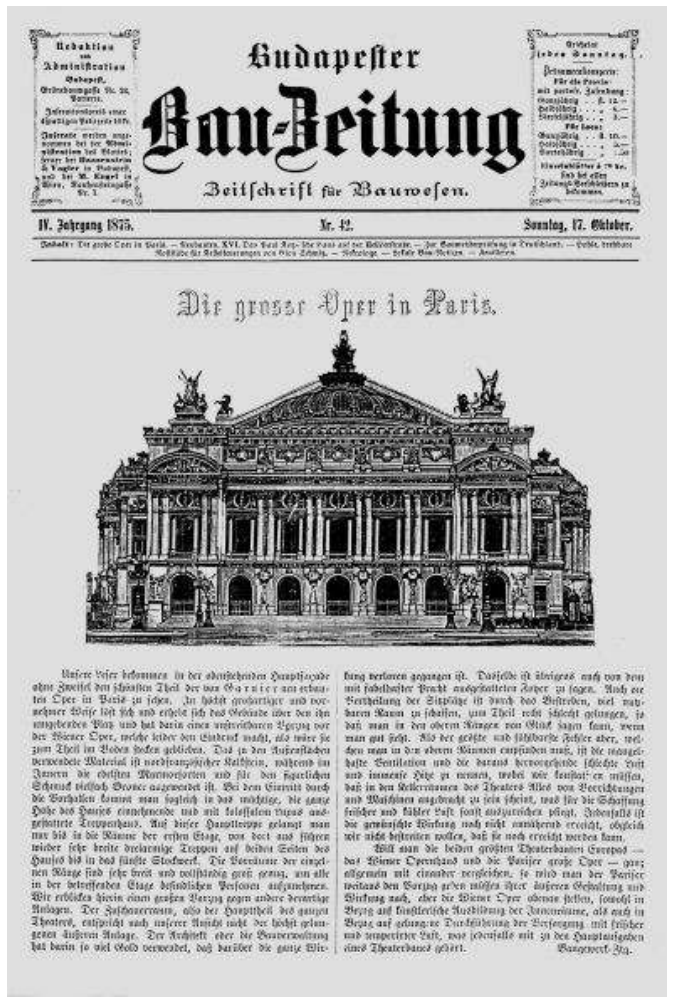

La majesté du grand escalier, la féerie créée par les miroirs, le déploiement de luxe dans le foyer, l'espace réservé à la scène reviennent le plus souvent comme motifs d'admiration. Un rédacteur du Baumeister (L'Architecte) publié à Berlin emploie même en 1903 à propos de l'Opéra cette expression devenue alors courante d'« œuvre d'art totale » pour saluer la réunion en un seul édifice de tous les arts décoratifs.

L'introduction de la mosaïque avec ses chatoyantes harmonies polychromes apparaît comme un choix très original de Garnier. La presse germanique salue cette profusion de couleurs et paraît avoir oublié le débat qui avait tant divisé les architectes dans la première moitié du XIXe siècle entre partisans et adversaires de la polychromie.

Dans le même quartier des grands travaux haussmanniens, les magasins du Printemps réédifiés par Paul Sédille à partir de 1883 -après un incendie-intéressent aussi particulièrement la presse germanique. Les innovations techniques de l'architecte qui a profité en deux occasions, pour le système de fondations et pour les piliers, des progrès de construction des grands ponts métalliques, offrent le sujet de deux articles à la Deutsche Bauzeitung en 1886. Plus tard, en 1915, un journal allemand, la Berliner Architekturwelt (Le Monde de l'architecture berlinois) rappellera que Paul Sédille a créé le premier grand magasin moderne et monumental qui a servi de modèle aux Allemands, avant que ceux-ci ne dépassent la France dans ce type de programme; Messel, par exemple, qui construit en 1896 le magasin Wertheim à Berlin. Les Allemands se distinguent particulièrement des autres pays d'Europe par l'attention qu'ils apportent aux règles de sécurité contre l'incendie. Victor Horta lui-même invité en 1903 à construire le Grand Bazar de Francfort se pliera avec soin à ces contraintes inhabituelles en Belgique, et y trouvera « un supplément d'originalité ». 
Sous la Troisième République, les rédacteurs de la presse germanique s'intéressent particulièrement à la construction publique à Paris, relevée de ses ruines après le siège des Prussiens et la Commune. La forme républicaine du régime engendre, selon la voix officielle du jeune Empire allemand, la Deutsche Bauzeitung, des œuvres moins somptueuses qu'une monarchie ne l'aurait fait. La critique se poursuit à propos de la province qui n'a pas su ou pas pu se défaire du carcan de l'École des beaux-arts. Mais ce jugement ne suffit pas à expliquer la rareté des articles sur les réalisations architecturales hors de Paris. La presse germanique reflète en ceci les principales revues d'architecture françaises, lesquelles offrent rarement un point de vue provincial, à quelques exceptions près : le nouvel hôpital du Havre de Jules Bourdais, l'église Notre-Dame-la-Treille à Lille de Jules-David Leroy objet d'un concours international, l'église Saint-Vincent-de-Paul à Marseille, l'église Saint-Pierre à Dijon de Jean-Baptiste Lassus, la nouvelle bourse de Dijon, le théâtre de Reims de Alphonse Gosset, la faculté de médecine de Toulouse de Lambert et la faculté de droit de Bordeaux édifiée par Jean Burguet dont la façade, lit-on dans la Deutsche Bauzeitung de 1887, est « harmonieuse et monumentale ».

26 Au tournant du siècle, le passage de la commande publique à la commande privée, phénomène que connaît alors la France, est nettement perçu dans la presse germanique, qui se fait l'écho d'une évolution semblable dans les pays allemands. La maison particulière, l'immeuble, les concours de façade à Paris deviennent un thème récurrent. Certaines revues se spécialisent dans ce programme d'un genre nouveau, telle l'Architektonische Rundschau, qui choisit de présenter des villas de style pittoresque, régional, normand, ou les Moderne Bauformen à la recherche, comme leur titre l'indique, des signes de renouveau architectural. Cette revue diffuse des planches d'encorbellements fantaisistes de Maxime Roisin, la gravure d'une maison japonisante de Henri Sauvage et Charles Sarazin (fig. 8 et 9) ou d'architectes moins connus comme Jean Desmoulins, Léon Benouville ou André Collin très apprécié pour ses maisons de campagne élégantes et confortables. 
Fig. 8 Maxime Roisin. Façade d'une maison et encorbellements de fer, Moderne Bauformen, 1902, pl. 9.

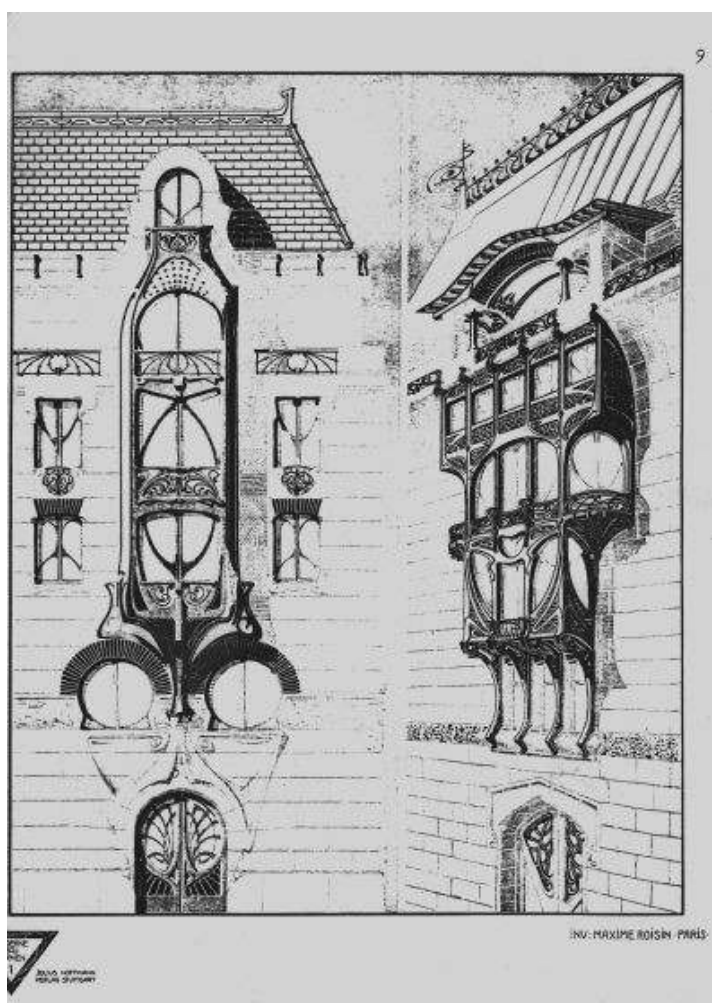

Fig. 9 Henri Sauvage et Charles Sarazin. Maison de campagne, Moderne Bauformen, 1902, pl. 9.

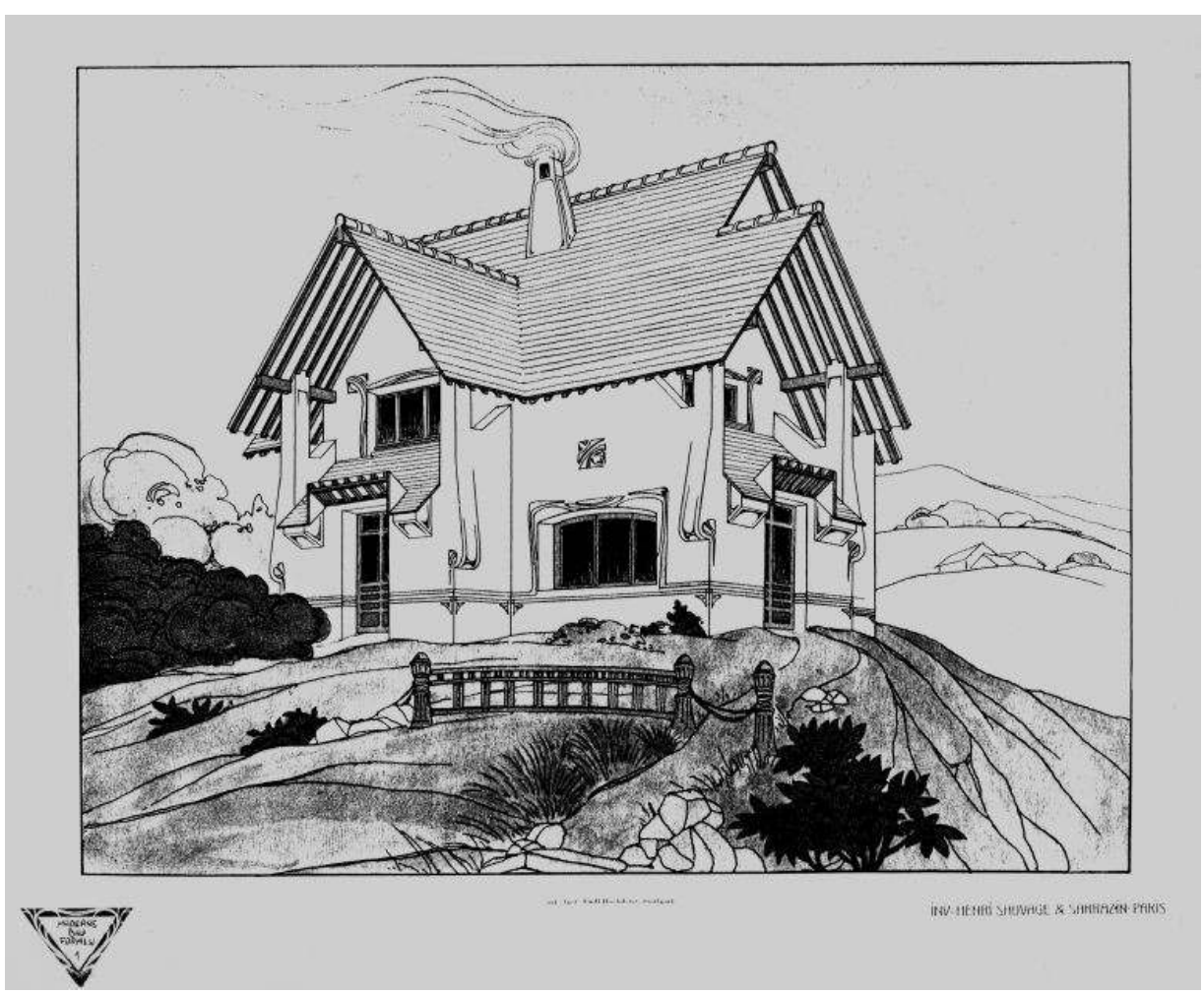

27 Les rédacteurs germaniques ont conscience que le relais de la modernité a été transmis de la France aux Belges et aux Allemands d'Europe Centrale. Les Français, convaincus 
de porter le bon goût à toutes les nations, doivent progressivement admettre, notamment à l'occasion des expositions universelles dont ils sont les hôtes et auxquelles ils participent systématiquement, qu'ils n'excellent plus dans les arts et qu'il est nécessaire de s'intéresser aux pays voisins. Les précurseurs français de l'architecture moderne comme Hector Guimard ou Robert Mallet-Stevens l'ont compris et se tournent, qui vers Victor Horta, qui vers Josef Hoffmann. Mais, malgré le caractère nationaliste des critiques qui remettent en cause la suprématie artistique de la France après 1870 , le ton des architectes reste mesuré, urbain, respectueux d'un pays dans lequel Jacques-Ignace Hittorff, Gottfried Semper, Otto Wagner, etc. ont profité de la rigueur de la formation académique.

\section{Liste des titres de revues d'architecture allemandes dépouillées avec leurs dates de parution}

Revues allemandes

- Architektonische Monatshefte, Stuttgart, 1900-1903 [exhaustif].

- Architektonische Rundschau, Stuttgart, 1885-1916 [1885-1886 ; 1895-1902].

- Die Architecktur des XX. Jahrhunderts, Leipzig, 1901-1914 [exhaustif].

- Bau- und Kunstgewerbe Zeitung für das deutsche Reich, Munich, 1869-1890 [1877 ; 1885-1890].

- Das Baugewerbe, Karlsruhe, 1885-1886 [1885-1886].

- Der Baumeister, Berlin, 1902-1944 [1903 ; 1914].

- Berliner Architekturwelt, Berlin, 1898-1919 [1899 ; 1913-1914].

- Centralblatt der Bauverwaltung, Berlin, 1881-1914 [sondages en 1902, 1905, 1906].

- Der Civilingenieur, Leipzig, 1853/55-1896 [1856].

- Dekorative Kunst [sondages en 1905-1907].

- Deutsche Bauhütte, Hanovre, 1897-1942 [1899-1900 ; 1914].

- Deutsche Bauzeitung, Berlin, 1867-1942 [1867-1914].

- Die Denkmalpflege, Berlin, 1899-1922 [sondages].

- Konkurrenz-Nachrichten, Leipzig, 1894-1910 [1905-1909].

- Moderne Bauformen, Stuttgart, 1902-1944 [1904-1914].

- Notizheft des Westpreussischen Architekten- und Ingenieur Vereins, Danzig, 1875-1881 [1875-1877].

- Der Rohrleger und Gesundheits- Ingenieur, Berlin, 1872-1879 [1878-1879].

- Der Städtebau, Berlin, 1904-1929 [1904-1914].

- Süddeutsche Bauzeitung, Munich, 1890-1922 [sondages en 1894, 1895, 1896, 1914].

- Technische Zeitschrift des Westpreussischen Architekten- und Ingenieur Vereins, Danzig, 1879-1881 [1879-1880].

- Wochenblatt für Baukunde, Francfort-sur-le-Main, 1878-1890 [sondages pour 1885-1890 grâce aux tables annuelles].

-Wochenschrift des Architektenvereins zu Berlin, Berlin, 1906-1922 [1906-1914].

-Zeitschrift des Architekten-und Ingenieurvereins zu Hannover, Hanovre, 1855-1895 [exhaustif].

- <\#ITALIQUES\#>Zeitschrift des Bayerischen Architekten - und Ingenieur-Vereines</ \#ITALIQUES\#>, Munich, 1869-1878 [1869-1873].

-Zeitschrift für Architecktur - und Ingenieurwesen, Hanovre, 1896-1923 [sondages en 1869, 1887, 1899-1900].

-Zeitschrift für Baukunde, Munich, 1878-1884 [sondages grâce aux tables annuelles pour 1878-1884]. 
- Zeitschrift für Bauwesen, Berlin, 1851-1944 [1872-1882].

- Zeitschrift für bildende Kunst, Leipzig, 1866-1932 [1866-1900].

- Zeitschrift für die Geschichte der Architecktur, 1908- [sondages en 1907-1913].

- <\#ITALIQUES\#>Zeitschrift für praktische Baukunst</\#ITALIQUES\#>, Leipzig, Berlin, 1841-1865 [sondages].

Revues de l'Empire austro-hongrois

- Allgemeine Bauzeitung, Vienne, 1836-1919 [1836-1914].

- Allgemeine Illustrirte Weltaustellungszeitung, parue pour l'exposition universelle de Vienne en 1873 [exhaustif].

- Der Architekt, Vienne, 1895-1922 [1895-1901].

- Architekten und Baumeister Zeitung, Vienne, Leipzig, 1898- [1899].

- Bauzeitung für Ungarn, Budapest, 1876-1896 [1876-1882]

- Chronik des Wiener Goethe-Vereins [1889].

- Der Civiltechniker, Vienne, 1879-1914 [1881].

- Mittheilungen der Kaiserlichen Königlichen Central-Commission zur Erfoschung und Erhaltung der Baudenkmale, Vienne, 1856-1861 [1856-1858].

-<\#ITALIQUES\#>Mittheilungen des Architekten-und Ingenieurvereins Böhmen</ \#ITALIQUES\#>, Prague, 1866-1882 [exhaustif].

- <\#ITALIQUES\#>Neue freie Presse </\#ITALIQUES\#>[1908].

- Österreichs Bau und Werkkunst [1924].

- Österreichisch-Ungarische Revue [1887].

- Pest-Ofner Bauzeitung, Pest, 1873-1876 [exhaustif].

- Technische Blätter, Prague, 1896-1921 [1896-1914].

- Ungarische Bauzeitung, Budapest, 1896-1914 [sondages en 1896-1897, 1914].

-<\#ITALIQUES\#>Wiener Bauindustriezeitung</\#ITALIQUES\#>, Vienne, 1883-1916/17 [1887-1897].

- <\#ITALIQUES\#>Zeitschrift des Österreichischen Ingenieur-Vereines</\#ITALIQUES\#>, Vienne, 1849-1865 [1851-1865].

-<\#ITALIQUES\#>Zeitschrift des Österreichischen Ingenieur- und Architekten- Vereines</ \#ITALIQUES\#>, Vienne, 1865-1938 [1865-1895].

\section{INDEX}

Index chronologique : XIXe siècle, XXe siècle, Epoque contemporaine Index géographique : Europe, Allemagne, France

Mots-clés : architecture, Allemagne, public spécialisé de constructeurs, équipement des villes, recherche de la modernité, francophobie, sécurité, polychromie, relais de la modernité, passéisme, expositions universelles 


\section{AUTEURS}

\section{ANNE GEORGEON-LISKENNE}

Conservateur du patrimoine au Ministère des Affaires Etrangères 